\title{
A Seasonal Cause of Encephalitis: Influenza Virus A (H3N1) Infection
}

\author{
Rúben Reis $^{1}$, Francisco Adragão², Catarina Parente ${ }^{1}$, Inês Nunes ${ }^{3}$, Armindo Ramos ${ }^{3}$, Inês Nabais ${ }^{4}$, Isa Silva ${ }^{4}$ \\ ${ }^{1}$ Internal Medicine Department, Centro Hospitalar Barreiro-Montijo, Barreiro, Portugal \\ ${ }^{2}$ Internal Medicine Department, Centro Hospitalar do Algarve, Algarve, Portugal \\ ${ }^{3}$ Intensive Care Unit, Hospital de Cascais, Cascais, Portugal \\ ${ }^{4}$ Internal Medicine Department, Hospital de Cascais, Cascais, Portugal
}

How to cite this article: Reis R, Adragão F, Parente C, Nunes I, Ramos A, Nabais I, Silva I. A seasonal cause of encephalitis: influenza virus A (H3N1) infection. EJCRIM 2021;8: doi:10.12890/2021_002336.

Conflicts of Interests: The Authors declare that there are no competing interests.

This article is licensed under a Commons Attribution Non-Commercial 4.0 License

\section{ABSTRACT}

Influenza virus infection can have a range of presentations, from asymptomatic to life-threatening disease. We present the case of a 57-yearold woman with a known history of schizophrenia (controlled with medication) who presented to our emergency department in a coma after experiencing a seizure. She had reported flu-like symptoms in the previous week, which evolved to dyspnoea and altered mental status culminating in seizures and coma. Influenza virus A (H3N1) was identified in the cerebrospinal fluid. Although a rare cause of encephalitis, the influenza virus should be considered in the differential diagnosis, especially during epidemics.

\section{LEARNING POINTS}

- Influenza virus encephalitis should be considered in some specific settings, such as during pandemics or seasonal epidemics.

- Altered mental status in a patient with a history of mental disease may be easily dismissed as secondary to prior disease or medication.

- Although rare, extrapulmonary manifestations of influenza virus infection can be serious and fatal.

\section{KEYWORDS}

Influenza A (H3N1), encephalitis, influenza-associated encephalopathy

\section{CASE DESCRIPTION}

The authors describe the case of a 57-year-old woman with history of hypertension, obesity, type 2 diabetes and schizophrenia (controlled with medication). The patient was chronically medicated with clozapine $200 \mathrm{mg} /$ day, biperiden $4 \mathrm{mg} /$ day, simvastatin $20 \mathrm{mg} / \mathrm{day}$, indapamide $1.5 \mathrm{mg} /$ day and metformin $1000 \mathrm{mg} /$ day.

The patient presented a week before admission with flu-like symptoms (fever, cough and malaise). Her mother also reported similar symptoms starting 1 week previously. There was no report of contact with animals (domestic or not), in particular pigs or live poultry, or recent travel abroad or to a rural area. The patient lived in a metropolitan area. On the day of her admission, she was brought to the emergency ward for dyspnoea and altered mental status. Her vital signs were: blood pressure of $90 / 50 \mathrm{mmHg}$, heart rate of 110 beats per minute, respiratory rate of 10 cycles per minute, oxygen peripheral saturation of $88 \%$, and tympanic temperature of $40.5^{\circ} \mathrm{C}$. Her physical examination revealed altered mental status with a Glasgow Coma Scale score of 13, crackles on the right hemithorax on auscultation and hemiplegia, with no other alterations at the time. Arterial blood gas was taken, which showed type 2 respiratory failure. 
Initial laboratory results are shown in Table 1. Influenza antigen was positive, and urine and blood tests were negative for benzodiazepine, ethanol and tricyclic antidepressants. A chest x-ray was compatible with right inferior lobe pneumonia, while computed tomography of the head was unremarkable, with no signs of ischaemic or haemorrhagic stroke, trauma or space-occupying lesions.

\begin{tabular}{|l|l|}
\hline Parameter & Value \\
\hline Haemoglobin & $14.5 \mathrm{~g} / \mathrm{dl}$ \\
\hline Leukocytes & $3,300 / \mathrm{mm}^{3}$ \\
\hline Neutrophils & $2,800 / \mathrm{mm}^{3}$ \\
\hline Lymphocytes & $123 / \mathrm{mm}^{3}$ \\
\hline Platelet count & $171,000 / \mathrm{mm}^{3}$ \\
\hline Serum creatinine & $2.04 \mathrm{mg} / \mathrm{dl}$ \\
\hline BUN & $81.4 \mathrm{mg} / \mathrm{dl}$ \\
\hline Influenza RT-PCR (blood sample) & Positive for influenza A H3N1 \\
\hline
\end{tabular}

Table 1. Initial laboratory results

\section{DISCUSSION}

Influenza most commonly presents as malaise, myalgia, fever and upper airway symptoms, but it can also present as pneumonia, myocarditis, acute renal failure, liver failure and encephalitis. Although a rare cause of encephalitis, the influenza virus should be considered in some specific settings, such as during its seasonal epidemics (winter) or in a pandemic setting (e.g., 2009 H1N1) ${ }^{[1]}$.

Influenza A (or swine flu) is a respiratory disease common among pigs and poultry. Influenza A appears to have been derived from viral ancestors in wild birds. It was transmitted from these wild birds through water and fomites to domestic ducks and poultry and thereafter to pigs, horses and cats. Humans can be infected through poultry and pigs by fomites, contaminated water and aerosols. Human-to-human infections occur mainly by respiratory droplets, aerosols or self- inoculation ${ }^{[2]}$. The most common variants in the wild are $\mathrm{H} 1 \mathrm{~N} 1$, $\mathrm{H} 1 \mathrm{~N} 2$, H2N1, H3N1, H3N2, and H2N3. In humans, the H3N1 and H1N1 (since the 2009 epidemic) are the most commonly identified variants. In this particular case, no other cases of the H3N1 variant were identified within our hospital, and there was no epidemiological follow-up. The patient's mother was not tested.

An outbreak was reported in a poultry farm in Belgium during this time which led to three different aviary outbreaks in northern France. No other outbreaks with the H3N1 variant in Europe were described at this time ${ }^{[3]}$.

The neurological complications of influenza A are described as influenza-associated encephalopathy (IAE), Guillain-Barré syndrome, Reye's syndrome and post-influenza syndrome ${ }^{[1]}$. Neurological complications are most frequently observed in younger patients ${ }^{[4]}$. Acute IAE is described as acute impairment of consciousness manifesting within the first few days of influenza infection ${ }^{[1,4,5]}$. In this particular case, the patient displayed an altered level of consciousness that progressed to a comatose state (GCS 3), hemiplegia and seizures. A review of IAE cases reported 28 cases, most of which were triggered by the H1N1 variant ${ }^{[1]}$. The presentation was heterogeneous in these reported cases, the most common symptom being a decreased level of consciousness (80\%), while other symptoms included seizure activity (37\%), urinary retention, hemiplegia and vision loss ${ }^{[1]}$. No case was found in the literature describing an influenza A H3N1 variant. The presence of viral RNA on CSF suggests direct viral invasion and allows the diagnosis of IAE ${ }^{[6]}$.

Initially, the neurological findings were interpreted either as secondary to our patient's sepsis or due to alterations caused by chronic medication. Only after progression to a comatose state with seizures and the identification of influenza A H3N1 in CSF, bronchoalveolar fluid and blood samples, was the diagnosis of IAE made. Therefore, it is of extreme importance to consider the central nervous system in patients with altered mental status even in the presence of sepsis or neurotropic medication. Unfortunately, there is not enough information about this variant to predict if its disease behaviour or prognosis differs from that of the H1N1 or H3N2 influenza A variants. 


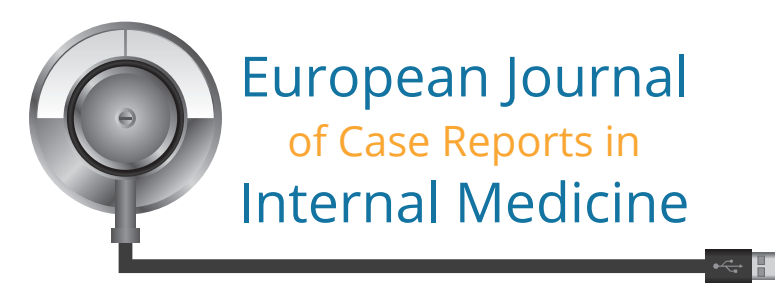

\section{REFERENCES}

1. Sellers SA, Hagan RS, Hayden FG, Fischer WA. The hidden burden of influenza: a review of the extra-pulmonary complications of influenza infection. Influenza Other Respir Viruses 2017;11(5):372-393.

2. Krammer F, Smith GJD, Fouchier RAM, Peiris M, Kedzierska K, Doherty PC, et al. Influenza. Nat Rev Dis Primer 2018;4(1):3.

3. European Food Safety Authority, European Centre for Disease Prevention and Control, European Union Reference Laboratory for Avian Influenza, Adlhoch C, Fusaro A, Kuiken T, et al. Avian influenza overview February-August 2019. EFSA J 2019;17(9):5843.

4. Meijer WJ, Linn FHH, Wensing AMJ, Leavis HL, van Riel D, GeurtsvanKessel CH, et al. Acute influenza virus-associated encephalitis and encephalopathy in adults: a challenging diagnosis. JMM Case Rep 2016;3(6):e005076.

5. Yuan H-T, Ho T-H, Lee J-T, Chen P-C, Wang C-W, Yang F-C. Simply influenza A (H3N2)-associated encephalitis with seizure. Am J Emerg Med 2019;37(9):1808.e1-1808.e3.

6. Fujimoto S, Kobayashi M, Uemura O, Iwasa M, Ando T, Katoh T, et al. PCR on cerebrospinal fluid to show influenza-associated acute encephalopathy or encephalitis. Lancet 1998;352(9131):873-875. 